\title{
Hygiène et soins du corps dans les couvents de femmes du Poitou au XVIIIe siècle
}

\section{Gwénael Murphy}

\section{(C) OpenEdition \\ 1 Journals}

Édition électronique

URL : http://journals.openedition.org/abpo/1373

DOI : $10.4000 /$ abpo.1373

ISBN : 978-2-7535-1491-1

ISSN : 2108-6443

\section{Éditeur}

Presses universitaires de Rennes

Édition imprimée

Date de publication : 20 octobre 2003

Pagination : 77-86

ISBN : 978-2-86847-929-7

ISSN : 0399-0826

\section{Référence électronique}

Gwénael Murphy, «Hygiène et soins du corps dans les couvents de femmes du Poitou au

XVIIle siècle », Annales de Bretagne et des Pays de l'Ouest [En ligne], 110-3 | 2003, mis en ligne le 20 décembre 2005, consulté le 21 avril 2019. URL : http://journals.openedition.org/abpo/1373 ; DOI : 10.4000/abpo. 1373 


\title{
Hygiène et soins du corps dans les couvents de femmes du Poitou au XVIII ${ }^{\mathrm{e}}$ siècle
}

\author{
Gwénaël MuRPHY \\ Docteur en histoire moderne (EHESS, Paris) - chercheur associé au CHRISCO \\ Professeur d'histoire au collège d'Airvault (Deux-Sèvres)
}

La question de l'hygiène et des soins du corps dans les couvents de femmes de l'époque moderne est mal connue ${ }^{1}$. L'influence de la doctrine chrétienne sur la " conduite corporelle " des religieuses a été étudiée pour le XIX ${ }^{\mathrm{e}}$ siècle, en particulier grâce aux travaux d'O. Arnold et J. Maître ${ }^{2}$. En revanche, l'observation de ces corps comme ceux de femmes ordinaires, l'inventaire des pratiques de santé et des formes de l'hygiène quotidienne dans les couvents des XVII ${ }^{\mathrm{e}}$ et XVIII ${ }^{\mathrm{e}}$ siècles reste à faire. Il n'y a rien de surprenant, en réalité, à cette situation, en particulier pour les religieuses. Celles-ci ont retenu l'attention des historiens par les soins qu'elles apportaient aux malades, insensés, vieillards, incurables, pauvres, militaires, vénériennes ou enfants abandonnés ${ }^{3}$. Ces femmes qui soignent les corps des autres font omettre qu'elles en possèdent également un, masqué sous des habits amples, un voile, une cornette... Le processus symbolique et vestimentaire consistant à " désexualiser " ces jeunes femmes lors de leur entrée en religion semble de ce fait parvenu à ses fins ${ }^{4}$.

1. Nous tenons à remercier particulièrement Annie Antoine, Sébastien Jahan et Pascal Hérault pour leurs conseils avisés concernant cette étude.

2. Particulièrement ARNOLD, Odile, Le Corps et l'âme. La vie des religieuses au XIXe siècle, Paris, Seuil, 1984, 373 p. et MAITRE, Jacques, Anorexies religieuses, anorexie mentale. Essai de psychanalyse sociohistorique. De Marie de l'Incarnation à Simone Weil, Paris, Cerf, 2000, 197 p.

3 . Sur les religieuses qui soignent, une abondante bibliographie dont on pourra retenir : Dervaux, Jeanne-Françoise, Le Doigt de Dieu. Les Filles de la Sagesse après la mort des fondateurs, t. I : 1759-1800, Cholet, Farré et Freulon, 1954, 348 p.; DinET-LECOMTE, MarieClaude, "Les religieuses hospitalières dans la France moderne : une même vocation dans une multitude d'instituts ", Revue d'Histoire de l'Église de France, t. LXXX, 1994, p. 195216; LEONARD Jacques, "Femmes, religion et médecine : les religieuses qui soignent en France au XIX ${ }^{\mathrm{e}}$ siècle ", Annales ESC, 32/4, sept.-oct. 1977, p. 887-907; VACHER Marguerite, Des "régulières » dans le siècle. Les sœurs de Saint-Joseph du Père Médaille aux XVII et XVII ${ }^{e}$ siècles, Clermont-Ferrand, Adose, 1991, 345 p.

4. De même les historiens de la santé sont-ils restés discrets sur les pratiques internes aux couvents : on note les silences de LEBRUn, François, Se soigner autrefois. Médecins, 
Autre difficulté, la rareté des sources qui ne sont pas disertes à ce propos. L'évocation des soins apportés à leur propre corps par les religieuses est toujours empreinte d'une inévitable pudeur. Pour cette étude, nous avons choisi une échelle d'observation régionale, le diocèse de Poitiers bien qu'il n'offre pas une quantité considérable de sources permettant d'étudier les pratiques d'hygiène et de santé dans les couvents de femmes d'Ancien Régime. En 1790, il compte cinquante-huit couvents ${ }^{5}$ parmi lesquels trois communautés seulement ont laissé des documents évoquant les soins corporels des religieuses : les fontevristes de Lencloître et de La Puye fondées au XII ${ }^{\mathrm{e}}$ siècle ${ }^{6}$ et les franciscaines de Posay-le-Vieil, fondées en 1641. Le croisement de ces sources permet toutefois d'essayer une modeste approche de la santé au couvent.

Notre étude se découpera en trois parties. L'inventaire des meubles des religieuses de Posay, lors de leur départ précipité en juillet 1791, permet de connaître les objets de toilette se trouvant dans chaque cellule à l'usage personnel des franciscaines. D'autre part, les fontevristes de La Puye ont laissé une règle intérieure spécifiant certaines obligations relatives à l'hygiène. Le troisième volet de ce travail utilisera la source la plus riche à propos des soins au couvent ${ }^{7}$ : le Livre des visites et opérations et remèdes que je fais aux dames religieuses de la communauté de Lencloître depuis le 12 juin 1786, rédigé par le chirurgien châtelleraudais Lafond et tenu quotidiennement pour les trois années 1786,1787 et $1790^{8}$. Ce type de document, en général resté dans les archives familiales des descendants de chirurgiens, permet de connaître avec précision toutes les opérations et types de soins apportés aux religieuses de Lencloître à la fin de l'Ancien Régime. À l'aide de ce document et de l'inventaire précis dressé quotidiennement par l'économe du prieuré des remèdes achetés à l'apothicaire Beaupoil, de Châtellerault, en 1787 et 1788, nous pouvons tenter de cerner les pratiques de santé au couvent à la fin de l'Ancien Régime.

\section{Hygiène de la cellule}

Les objets inventoriés en 1791 dans les cellules des religieuses de Posayle-Vieil comprennent de nombreux éléments relatifs à l'hygiène personnelle.

saints et sorciers aux XVIT et XVII e siècles, Paris, Seuil éd. 1995, 202 p. ou de VigARELLO, Georges, Le Propre et le sale. L'hygiène du corps depuis le Moyen Âge, Paris, Seuil, 1985, 282 p. et Histoire des pratiques de santé. Le sain et le malsain depuis le Moyen Âge, Paris, Seuil, 1993, 390 p. Voir notre article " Hygiène et pratiques de santé dans les couvents de femmes du Châtelleraudais au XVIII ${ }^{\mathrm{e}}$ siècle ", Revue d'Histoire du Pays Châtelleraudais n ${ }^{\circ}$ 4, $2^{\mathrm{e}}$ sem. 2002, p. 113-131.

5. Selon Archives Nationales, D XIX/6-106 à 109 : Inventaire du Comité Ecclésiastique de l'Assemblée Constituante de 1789. État des religieuses en 1790, diocèse de Poitiers.

6. À leur propos : Arch. dép. de la Vienne, 2 H 5/18 : Couvent de Lencloître, 1374-1777; 2 H 5/25 et 26 : Couvent de La Puye, 1493-1774.

7. Aucun document similaire n'existe pour les autres couvents de femmes du diocèse de Poitiers.

8. Arch. dép. de la Vienne, L 252 : Séquestre des biens, traitements et pensions des clergés séculier et régulier du canton de Saint-Genest, 1790-an VIII. 
Pourtant, au XVIII ${ }^{\mathrm{e}}$ siècle, les corps sont souvent sales, comme l'est l'environnement ${ }^{9}$. La présence d'une baignoire et de quelques bassines ( 5 pour treize religieuses) démontre que la mode des bains, disparue aux $\mathrm{XvI}^{\mathrm{e}}$ et $\mathrm{XVII}^{\mathrm{e}}$ siècles par crainte de la syphilis, réapparaît même dans les couvents ${ }^{10}$. Quoique peu chère, une seule baignoire est présente, dans la cellule de Marie de Castaldi, une franciscaine âgée de soixante-douze ans issue d'une famille fortunée ${ }^{11}$. La difficulté d'approvisionnement en eau ne saurait expliquer cette rareté, le couvent se situant en bord de Creuse. Cependant, peutêtre faut-il considérer la portée symbolique de l'eau. Son usage devient au $\mathrm{XVIII}^{\mathrm{e}}$ siècle une marque nouvelle de distinction sociale, en même temps qu'il correspond à une conception nouvelle de l'hygiène, l'immersion du corps n'étant plus considérée comme néfaste à la santé ${ }^{12}$. Prendre un bain est moins un simple geste de propreté qu'un plaisir de riche où se mêlent tout à la fois raffinement et sensualité : n'oublions pas que les cellules ne peuvent être verrouillées, et le risque d'être surprise nue s'avère probablement suffisamment dissuasif pour les religieuses pour expliquer l'absence de baignoire.

L'abondance de linge laisse supposer qu'elles procèdent à une " toilette sèche " ainsi qu'on le nomme à l'époque, soit un changement de vêtements très fréquents qui évitent la toilette. Ne pas toucher son corps est un interdit fort dans les règles religieuses, en particulier les parties sexuelles ${ }^{13}$. Aussi l'hygiène corporelle est-elle remplacée au couvent par l'hygiène vestimentaire. Pourtant, la présence de savons (deux par religieuse), de cuvettes (31) et de bassins laisse présager que ces règles sévères pliaient en pratique devant la nécessité, du moins la possibilité d'évacuer fréquemment les odeurs corporelles indésirables que l'absence de parfums empêche de masquer. Chaque religieuse possède dans sa cellule une bassine et un savon : c'est le minimum hygiénique conventuel. Alors que les miroirs sont bannis, par refus de la coquetterie apparentée à la futilité mondaine, curieusement, les peignes sont relativement nombreux (12). La présence des pots de chambre n'est pas à négliger (21). À proximité de la rivière, elle confirme que la toilette et l'hygiène ont été individualisées afin d'éviter tout contact visuel entre les corps des franciscaines.

9. GARnot, Benoît, La Culture matérielle en France aux XVIe, XVII, XVIII ${ }^{e}$ siècles, Paris, Ophrys, 1995; évoque cette " hygiène autre ", p. 125-133.

10. LATY, Dominique, Histoire des bains, Paris, PUF, 1996, p. 47-49.

11. Arch. dép. de la Vienne, 2 H 5/56 : lors de son entrée au couvent, son père, procureur du Roi à Montmorillon, verse en 1740 plus de quatre mille livres à la communauté de Posay.

12. GARNOT, Benoît, op. cit., p. 127.

13. Voir entre autres: Constitutions des Bénédictines de la congrégation du Calvaire, Paris, Coignard, 1635, p. 89-92; Constitution des religieuses de la Visitation, Paris, Coignard, 1676, p. 111; Règle et constitutions de l'ordre de Fontevrault, Paris, 1642, p. 78-81; Règles communes des Filles de Notre-Dame, Bordeaux, 1642, p. 234; Règlement pour les religieuses de Sainte-Ursule, Paris, 1676, p. 300-308. 
Ce bilan matériel laisse supposer, chez les franciscaines de Posay, une toilette fréquente et une hygiène respectable, sans nul doute plus élaborée que la norme du $\mathrm{XVIII}^{\mathrm{e}}$ siècle. En cela, elles se rapprochent des pratiques nobiliaires, catégorie sociale dont huit des treize professes présentes en 1791 sont issues. La tradition familiale a donc franchi le cloître, les pratiques sociales s'y retrouvent en dépit des règles religieuses. Le cloître reflet du monde qui l'entoure, du moins de celui d'où viennent les cloîtrées, telle est l'image à retenir de l'inventaire des objets ayant trait à l'hygiène personnelle dans le couvent de Posay.

Bien entendu, cet inventaire des treize cellules occupées du couvent de Posay ne laisse pas présager des pratiques réelles. Faute de témoignage, on ne peut savoir si Magdelaine Delanoue, qui possède un savon, un peigne, une bassine, une cuvette et un pot de chambre les utilise fréquemment, occasionnellement ou jamais, si elle est d'une propreté irréprochable, régulière, irrégulière ou d'une saleté repoussante. Le constat intéressant, ici, réside dans le fait que cet inventaire laisse présager que la vie religieuse ne régit plus l'intime et le corporel au-delà de chaque porte de cellule, et que les objets nécessaires à la toilette personnelle du XVIII ${ }^{\mathrm{e}}$ siècle sont mis à la disposition des religieuses.

\section{Règlement du corporel}

La règle de vie manuscrite des fontevristes de La Puye ne contredit pas le constat précédent ${ }^{14}$. L'image idéale du corps forgée par les règles religieuses s'apparente à une aspiration vers la perfection. Rédigée dans les années 1760, elle permet de connaître le modèle vers lequel les fontevristes de La Puye sont poussées.

Les sœurs doivent se coucher " vêtues et ceintes, c'est-à-dire qu'elles portent l'habit, une ceinture de crin sur la tête, un petit voile noir doublé d'une bande de voile blanc non cousu ou voile noir ${ }^{15}$ ". La couche elle-même se révèle fort sommaire, deux tréteaux sur lesquels reposent trois planches supportant une paillasse et des couvertures de laine, sans draps. La volonté est nettement de contrôler la nuit, espace redouté car lieu des tentations corporelles : sans le dire, le fait de garder l'habit de religieuse la nuit ne viset-il pas à empêcher et à culpabiliser psychologiquement les professes qui verseraient dans l'onanisme, strictement interdit et réprimé par l'Église ${ }^{16}$ ? Il ressort une volonté de " moraliser l'ombre " en " abolissant la nuit ${ }^{17}$ ".

14. Arch. dép. de la Vienne, 2 H 5/26, datée de 1768, l'auteur n'est pas connu. Ce genre d'additifs internes à la règle est fréquent dans les communautés religieuses, ajustement local de statuts généraux (voir pour Poitiers Règle des filles religieuses de l'ordre de SaintBenoît pour le monastère de Sainte-Croix, Poitiers, Thoreau 1612, 400 p. ou Les constitutions de la congrégation des religieuses hospitalières de Saint-Joseph de Poitiers, Paris 1686, 198 p.).

15. Sauf mention contraire, le reste du paragraphe se base sur Arch. dép. de la Vienne, 2 H5/26.

16. Foucault, Michel, Les anormaux. Cours au Collège de France, 1974-1975, Paris, Seuil/Gallimard, collection " Hautes Études ", p. 123-136 su l'onanisme.

17. Expressions empruntées à DELATTRE, Simone, Les douze heures noires. La nuit Paris au XIX siècle, Paris, Albin Michel, 2000, p. 111 et 411. 
Pour le jour, la tenue vestimentaire est bien entendu très réglementée, encore qu'une phrase laisse présager que l'adaptation selon les lieux et les temps de l'année est permise : "Chacun se couvre selon son besoin. " Le costume s'avère relativement complexe. Les sous-vêtements consistent en une tunique de serge blanche qui descend en dessous des reins, surmontant un jupon de toile. Par-dessus, l'habit est de bure noire, ceinture de crin, scapulaire peu échancré, surmonté d'une guimpe de toile blanche, et un bandeau de toile blanche cache le front. Un voile noir supplémentaire recouvre entièrement le visage lorsque les fontevristes se trouvent à l'office, aux prédications et au parloir, soit en tout lieu où les regards d'hommes, religieux (prêtres ou moines) ou laïcs (familles, paroissiens) peuvent se poser sur elles. Le même paragraphe contient deux lignes qui confirment l'indépendance des soins du corps repérés à Posay-le-Vieil : " Chaque religieuse a des objets personnels de toilette, peigne, brosse, etc., une cruche et un plat de terre servant de cuvette. " Le " etc. " laisse insatisfait, mais l'on suppose aisément qu'il suggère les savons et pots de chambre. Dans une règle fontevriste, sévère, inspirée de celle de saint Benoît qui régit l'ensemble de la vie religieuse et intime des sujets des monastères, le souci de l'hygiène l'emporte sur la tradition. Le XVIII ${ }^{\mathrm{e}}$ siècle, théâtre des premières manifestations hygiénistes et de souci de propreté corporelle par l'eau ${ }^{18}$, laisse transparaître ces soucis intimes au couvent. L'éducation de ces filles issues de familles aisées (17\% de nobles et $25 \%$ de filles d'hommes et de lois et de finances), qui sont les professes auxquels les pouvoirs conventuels (supériorat, économat, conseil) sont confiés, influe probablement sur cette évolution. Habituées à une hygiène personnelle et intime élaborée au sein de leurs familles, les religieuses de la fin de l'Ancien Régime refusent de céder sur ce point à la vie monastique. L'intime parvient à exister dans les couvents de femmes.

Enfin, il semble que la maladie ne soit pas, au contraire d'autres communautés, glorifiée comme souffrance à Dieu ${ }^{19}$. Dans la règle de La Puye, il est mentionné que "rien n'est épargné pour procurer la guérison ou le soulagement des malades", ce que confirme d'autres documents. La déclaration de revenus et dépenses au clergé de France de 1729 révèle 120 livres de charges " pour les médecins et chirurgiens ${ }^{20}$ ", et le contrat de religion de Marie Lignaux, en 1701, mentionne qu'en contrepartie des deux cent cinquante livres annuelles que versera son père, le marquis de Tussac, au couvent, les religieuses s'engagent à lui fournir " nourriture, entretien, vêtements nécessaires à son état tant en santé que maladie ${ }^{21}$ ". Alors que l'in-

18. VIGARELLO, Georges, op. cit., p. 141-195 en particulier.

19. C'est le cas aux Filles de Notre-Dame de Poitiers ou au Calvaire de la même ville qui refusent ostensiblement d'apporter les soins chirurgicaux ou médicaux nécessaires à leurs religieuses malades (voir Arch. dép. de la Vienne, registre 298 : Filles de Notre-Dame de Poitiers, vêtures et sépultures, 1618-1789 et Archives de l'ordre du Calvaire, carton 32 : Documents antérieurs à la Révolution).

20. Arch. dép. de la Vienne, G 422 : Déclarations des états de charges et de revenus des communautés religieuses du diocèse de Poitiers, 1729.

21. Arch. dép. de la Vienne, 2 H 5/26 : Fontevristes de La Puye, 1704-1774. 
timité et la toilette corporelle semblent laissées à l'appréciation de chaque religieuse, et de ce fait échappent au contrôle permanent induit par la règle, les soins extérieurs dispensés par médecins et chirurgiens se diffusent. Pour le Poitou, les mêmes reçus et quittances se retrouvent pour toutes les communautés de femmes : dès 1668, les bénédictines de Sainte-Croix de Poitiers, par exemple, déclarent 60 livres annuelles pour les « médecins et drogues ", et en 1790 elles payent pour le même poste de dépenses 90 livres $^{22}$.

\section{La religieuse, le chirurgien et l'apothicaire}

L'exemple le plus probant sur les soins apportés par un chirurgien à des religieuses concerne le couvent des fontevristes de Lencloître, dans le nord du diocèse. La méticulosité du chirurgien Lafond dans la tenue de son "Livre des visites que je fais aux dames religieuses de la communauté de Lencloître " n'a d'égale que la fréquence de ces mêmes visites. Résidant dans le bourg où est situé le couvent en question, il n'épargne pas ses déplacements et appartient à ces rares laïques qui franchissent presque chaque jour les portes d'un cloître de femmes. Il est vrai que la pudeur des religieuses ne s'applique peut-être pas aux fontevristes, ordre mixte dans sa fondation où les femmes ont même la supériorité hiérarchique et canonique sur les hommes ${ }^{23}$. Mais les déplacements du chirurgien ne relèvent pas de la charité, puisque, en dépit du silence que fera Radegonde Bourbeau, la prieure, à son sujet en déclarant les charges du monastère en $1790^{24}$, ce sont plus 250 livres qui lui sont versées chaque année.

L'été semble la période la plus propice aux problèmes de santé au couvent : les mois de juin à septembre sont ceux où ont lieu le plus grand nombre de visites. Cette périodicité ne correspond pas, à titre de comparaison, avec le rythme repéré par P. Hérault à l'Hôtel-Dieu de Montmorillon, aux confins méridionaux du diocèse. Selon le livre des remèdes de Jean Rozet, moine Augustin et chirurgien de l'hospice, il y a " une activité mensuelle constante de 35 prescriptions par mois [...] l'ampleur maximale est atteinte en mars et avril [...] l'afflux printanier correspond à l'époque de la soudure ou les organismes s'affaiblissent et l'activité microbienne est stimulée $^{25}$ ". Mais plus que la fréquence, c'est la nature de ces visites et la

22. Arch. dép. de la Vienne, 2 H 5/69 : Religieuses de Notre-Dame de Châtellerault, rentes, constitutions, installation, quittances (1640-1789) et L 241 : Canton de Châtellerault, clergé (1790-an VIII).

23. Rappelons que l'ordre de Fontevrault est dirigé par l'abbesse du même nom. Sur ces particularités, voir les travaux de DALARUN, Jacques, L'impossible sainteté. La vie retrouvée de Robert d'Arbrissel (vers 1045-1116), Paris, Cerf 1985, 382 p. et " Robert d'Arbrissel et le salut des femmes " dans Moines et religieux au Moyen Âge, Paris, Seuil, 1994, p. 29-45.

24. Arch. dép. de la Vienne, 2 H 5/18 : Couvent de Lencloître, 1432-1790.

25. HERAult, Pascal, "Soigner en Montmorillonnais au début du XVIII ${ }^{\mathrm{e}}$ siècle (d'après le livre des remèdes de l'Augustin Jean Rozet) ", Annales de Bretagne et des Pays de l'Ouest, t. 100, 1993/1, p. 61-120 (ici p. 95-96). L'apothicaire de la Maison-Dieu de Montmorillon traite des patients fort différents des religieuses, ce qui explique cette différenciation. 
façon dont elles sont retranscrites par le chirurgien, les mots employés, qui retiennent l'attention.

La saignée est l'opération la plus courante, précédée en général par plusieurs séances de pose de sangsues, expliquant la corrélation quantitative entre ces deux types d'intervention. Le $1^{\text {er }}$ juillet 1786 , Lafond procède ainsi à une saignée au pied de la sœur Radegonde. Le lendemain, il revient procéder à la même opération sur les sœurs Aimée de James, apothicaire du couvent et de Lugnies. Le 9, il tire une dent " pourrie et déchaussée " à la religieuse Preneuf. La plupart du temps, une visite de contrôle suit la saignée, comme c'est le cas pour la sœur Minotier, qui voit Lafond lui ouvrir le bras le 16 août de la même année puis s'assurer quotidiennement durant une semaine du bon rétablissement de la malade. La visite en question vaudra une livre seulement à l'économe, la saignée en revanche double les tarifs. L'absence complète de mention d'un médecin laisse présager que Lafond remplit également sa tâche, le diagnostic, qu'il pose lui-même sur la professe souffrante : "Le diagnostic précède la thérapeutique ${ }^{26}$. "

Tirer le sang rassure les malades en dépit des dérives dénoncées dans les facultés de médecine ${ }^{27}$. Deux moyens essentiels sont employés : la pose de sangsues (67 mentions), parfois en grand nombre (pas moins de vingtdeux dans le dos de la sœur Arnault le 2 janvier 1788, sept dans le cou de la sœur de James trois mois plus tard) et la saignée (50 mentions), qui demeure la médication essentielle dans la France du XvIII ${ }^{\mathrm{e}}$ siècle. Elle consiste en une " opération de chirurgie qu'on fait avec une lancette pour tirer le sang corrompu ou superflu qui est dans les veines ${ }^{28}$ ". Le principe selon lequel plus on tire de l'eau d'un puits et plus elle est bonne, joint aux croyances de la contenance liquide du corps qui consisterait en vingt-quatre litres de sang dont vingt peuvent être retirés sans mourir, expliquent sans doute son incroyable fortune. Au couvent de Lencloître comme ailleurs, elle s'avère un moyen curatif ordinaire. Lorsque le 12 décembre 1787 Lafond saigne la sœur Ayrault par les veines du pied, elle souffre probablement d'hémorroïdes ${ }^{29}$. La religieuse Mitonnier, saignée au bras en août précédent, se plaint sans doute du foie, alors que ses compagnes Pulzereau, ouverte au bout du nez et aux angles internes des yeux le 14 juin 1788, et Gourdon, ouverte sur la veine sciatique le 6 juillet suivant, souffraient la première de maux de tête et la seconde de la goutte. La veine ouverte est toujours proche du mal, mais jamais in situ. L'opération n'est pas sans danger. Adélaïde Gourdon manifeste dès le 7 juillet, lendemain de l'opération, un hématome à l'emplacement de la piqûre. Peu à peu, l'abcès enfle et le

26. LEBRUN, François, op. cit., p. 59.

27. Ibidem, p. 62-67 sur les saignées et les purgatifs. On pourra consulter également à ce sujet LEBIGRE, Arlette, « Et saigner et purger! ", L'Histoire n ${ }^{\circ}$ 74, janvier 1985, p. 110-113; LEONARD, Jacques, « À propos de l'histoire de la saignée (1600-1900) », Mentalités n 1, 1988, p. 75-89; Molitor-Canasevio B., "Saignée ", Bluche, François (dir.), Dictionnaire du Grand Siècle, Paris, Fayard, 1990, p. 1376-1377.

28. LEONARD, Jacques, "À propos de l'histoire de la saignée ", op. cit., p. 78.

29. Arch. dép. de la Vienne, 2 H 5/18 pour cette opération et les suivantes. 
13, " elle était saisie de grands frissons, alors que l'abcès avait recouvert l'épaule et gagnait le coffre ". Elle trépasse trois jours plus tard : la saignée s'est révélée, comme trop souvent, mortelle. Certaines survivent toutefois, comme la sœur de James, saignée seize fois en quatre mois en 1787.

En dehors de cette intervention courante, le travail de Lafond auprès des religieuses se révèle varié. Il prescrit fréquemment des purgatifs (33 fois), mélanges de médicaments et d'herbes aboutissant volontairement à des vomissements et des diarrhées censées purger le corps de la malade : ainsi, du 6 au 17 août 1786, vient-il visiter à vingt reprises la sœur Gilliette (parfois le matin et l'après-midi du même jour) en lui prescrivant à chaque fois des " purgatifs ". Le 22 septembre, on fait appel à lui " de nuit, afin de faire ingérer de toute urgence un bol purgatif à la sœur Arnault ". Certaines religieuses sont ainsi fort fragiles. Aimée de James, chargée des médicaments du couvent et dépassant tout juste la trentaine, reçoit à elle seule un quart des visites de Lafond en 1786. La présence de deux ou trois tiers à chaque visite exclut toute relation intime entre les deux personnes, sensiblement d'âge égal ${ }^{30}$. Saignées, sangsues dans le cou et sur les jambes, purgatifs et surtout " calmants ", "pilules pour dormir " et "somnifères " sont prescrits incessamment pour cette professe dont le problème essentiel semble résider en une insomnie chronique qui entraîne fatigue et fragilité physique excessive. Parfois son rôle consiste également en l'accompagnement à la mort d'une religieuse âgée. Radegonde Lebeau, née en 1710 et entrée au couvent en 1730, reçoit sa visite presque chaque jour entre le 11 juillet et le 6 septembre 1788, veille de son décès. Les remèdes prescrits se limitent à quelques pilules et deux poses de sangsues (dont l'une, seule du genre, sur les tempes, censée contrer les maux de tête). Il s'agit de soulager au mieux une femme incurable en fin de vie ${ }^{31}$. Par deux fois, enfin, il procède " à une opération " sur les sœurs Lavallée le 12 septembre 1790 et Lugniers le 15 novembre suivant. Si la première qui semble fort mal en point puisqu'il la visite quotidiennement durant les trois mois qui suivent, survit, la seconde intervention échoue et la sœur Lugniers meurt deux jours plus tard. La nature des opérations n'est malheureusement pas mentionnée dans le registre du chirurgien.

Les relations que fait Lafont de son activité, destinées seulement à calculer ses honoraires, sont fort sommaires. Il manque, en particulier, la pathologie des malades. En revanche, le vocabulaire qu'il emploie à propos des religieuses reflète une compréhension fine de l'organisation du couvent. Lorsqu'il visite l'une d'entre elle occupant une fonction, celle-ci ne manque jamais d'être mentionnée : "la dame Bourbeau, prieure ", " la sœur Arnault, conseillère " ou la " sœur de James, apothicaire ". En revanche, la fonction disparaît si elle n'est pas jugée d'importance : pas de cuisinière, de portière ou de lingère mentionnée à côté de noms restés seuls

30. Selon son propre registre, Lafond a trente-neuf ans en 1788, soit six de plus qu'Aimée de James.

31. Arch. dép. de la Vienne, 2 H 5/18 pour l'acte de sépulture. 
sur le registre. Enfin, par cinq fois, il va simplement apporter des soins à " une postulante ". N'ayant pas encore le statut de religieuse, son nom même ne présente pas d'intérêt à l'esprit de Lafond. Inconsciemment, il respecte dans ses retranscriptions la hiérarchie conventuelle, admise, comme nous le constatons, connue et normalisée dans les mentalités d'Ancien Régime.

Mais l'intervention du chirurgien ne se révèle pas toujours nécessaire. Aussi prescrit-il le plus souvent des compositions liquides à introduire dans les corps par voie anale ou buccale qui fait intervenir dans des proportions variables de multiples produits de la pharmacopée de l'époque. L'apothicaire de Lencloître, Aimée de James, possède son local des pots, boîtes cylindriques et chevrettes, bouteilles et bocaux de verre portant le nom latin du produit contenu ${ }^{32}$. Méticuleuse, la sœur tient elle aussi un livre des remèdes achetés à l'apothicaire Beaupoil, de Châtellerault, entre 1787 et 1790, à hauteur de soixante par an environ pour une somme considérable qui avoisine les 400 livres ${ }^{33}$.

Selon le registre de l'année 1786, les potions, remèdes et médicaments les plus recommandés et achetés sont les suivants : réglisse, sirops et quinquina, suivis des fleurs d'orange, de l'huile d'amande douce et des têtes de chenilles. Ces dernières, très nombreuses dans les achats de l'apothicaire du couvent, sont recherchées pour leurs têtes, jugées efficaces contre les ulcères, les rhumatismes et la goutte ${ }^{34}$. L'achat de sangsues, de même que l'existence d'un jardin botanique dans lequel l'apothicaire du couvent se servait pour préparer elle-même certains remèdes ${ }^{35}$, démontre que l'automédication fut largement pratiquée au couvent. L'intervention du chirurgien Lafond, tout comme les achats à l'apothicaire Beaupoil, pallient le manque de sources directement issues des religieuses à ce sujet. L'apothicaire de la communauté elle-même, la sœur Aimée de James, si souvent malade, n'évoque pas une seule fois dans sa correspondance privée conservée (la seule du genre dans le diocèse de Poitiers) son travail de soins et de préparation de remèdes ${ }^{36}$.

32. À propos de cette religieuse, voir notre article " Aimée de James, religieuse de Lencloître (1755-1794) ", Revue d'Histoire du Pays Châtelleraudais n ${ }^{\circ} 3,1^{\mathrm{er}}$ sem. 2002, p. 44-88.

33. Arch. dép. de la Vienne, L 252 : Séquestre des biens, pensions et traitements des clergés séculier et régulier, canton de Saint-Genest, 1790-an VIII où se trouvent les "Comptes de paiement des remèdes achetés à l'apothicaire Beaupoil de Châtellerault depuis cette année 1787 par Aimée de James, apothicaire des religieuses fontevristes du monastère de l'Encloître-les-Girondes".

34. Selon LEONARD, Jacques, "La médecine populaire en France au XIX ${ }^{\mathrm{e}}$ siècle ", Revue d'Histoire Moderne et Contemporaine, 1980, p. 510-516.

35. Arch. dép. de la Vienne, L 252 : Séquestre des biens des clergés séculier et régulier, canton de Saint-Genest d'Ambière, couvent des fontevristes de Lencloître (1790-an VIII).

36. Archives Nationales, W/09-303/345 : Juridictions extraordinaires, Tribunal Révolutionnaire. Procès Aimée de James. Cette correspondance avec sa nièce a été étudiée en détail dans notre travail Femmes de Dieu et Révolution Française dans le diocèse de Poitiers, thèse de doctorat, EHESS, Paris, 2003, $880 \mathrm{f}$. 
Mais les cinq livres de comptes tenus par Lafond et pour le paiement des remèdes n'en demeurent pas moins exceptionnels : aucun document comparable n'existe pour une autre communauté religieuse poitevine. Avec l'inventaire du mobilier cellulaire des franciscaines de Posay-le-Vieil et aux règles de vie des fontevristes de La Puye, il nous fourni un aperçu certes incomplet mais néanmoins signifiant, de l'hygiène et des pratiques de santé dans les couvents de femmes du Poitou au XVIII ${ }^{\mathrm{e}}$ siècle. Le mythe de corps tabous et intouchables, la croyance selon laquelle les religieuses ne se lavaient pas ne sont qu'un souvenir à la fin de l'Ancien Régime. Au sein de ces communautés contemplatives, nul doute que la toilette personnelle s'avère autorisée, voire recommandée, qu'une certaine intimité corporelle existe pour chaque professe et que les malades ne sont pas abandonnées aux soins arbitraires d'infirmières religieuses au savoir empirique et peu sûr. Le bassin et le savon font partie du mobilier commun de chaque sœur, au même titre que le lit ou le prie-Dieu, le chirurgien est un familier du couvent, un homme de confiance autorisé soigner les corps comme l'aumônier et le confesseur se doivent d'avoir soin des âmes. Reflets des origines sociales aisées des religieuses, ces pratiques démontrent, d'une certaine façon, que le cloître est à l'image du monde.

\section{RESUME}

Si de nombreuses religieuses soignent les corps des pauvres et des malades à l'époque moderne, les soins qu'elles apportent à leurs propres corps demeurent mal connus. Trois couvents du diocèse de Poitiers permettent, grâce à leurs archives, de lever une partie du voile qui recouvre les pratiques de santé des religieuses au $\mathrm{XVIII}^{\mathrm{e}}$ siècle. Les objets autorisés pour la toilette personnelle de ces femmes, retrouvés dans leurs cellules lors des inventaires de 1791-1792, permettent d'avoir un aperçu sur l'hygiène intime au couvent. Une règle intérieure spécifique au couvent de contemplatives de La Puye définit la théorie des soins et de l'hygiène personnelle autorisés. Enfin, le livre de compte d'un chirurgien de Châtellerault, chargé de soigner les religieuses d'un couvent fontevriste voisin, offre un point de vue inédit sur trois années (1786-1788) des opérations, médicaments et diverses interventions, presque quotidiennes, que sollicitent ces femmes pour protéger leur santé.

\section{ABSTRACT}

If a lot of nuns treat bodies of poors and ills at the modern time, the way they took good care of their bodies stay unrecognized. Three convents of Poitiers's diocese allow, in favour of their archives, to remove the veil on the health of nuns in $X V I I I^{\text {th }}$ century. The toilet requisites which were authorized in their cells, found during the inventories of 1791-1792, permit to have a general idea of private hygiene in convent. A specific rule in the contemplation convent of La Puye defines the theory of hygiene and health authorized. Lastly, the book of counts of a Chatellerault's surgeon, who treated the nuns of Lencloitre's convent, offer an original point of vue on three years (1786-1788) of operations and drugs, almost everyday, who were seeked by these women for the protection of their health. 